\title{
Article
}

\section{Study of inequalities for unified integral operators of generalized convex functions}

\author{
G. Farid ${ }^{1}$, K. Mahreen ${ }^{1}$ and Yu-Ming $\mathrm{Chu}^{2,3, *}$ \\ 1 Department of mathematics, COMSATS University Islamabad, Attock Campus, Pakistan. \\ 2 Department of Mathematics, Huzhou University, Huzhou 313000, P. R. China. \\ 3 Hunan Provincial Key Laboratory of Mathematical Modeling and Analysis in Engineering, Changsha University of \\ Science \& Technology, Changsha 410114, P. R. China. \\ * Correspondence: chuyuming@zjhu.edu.cn
}

Academic Editor: Emrah Evren Kara

Received: 1 December 2020; Accepted: 2 January 2021; Published: 20 February 2021.

\begin{abstract}
The aim of this paper is to study unified integral operators for generalized convex functions namely $(\alpha, h-m)$-convex functions. We obtained upper as well as lower bounds of these integral operators in diverse forms. The results simultaneously hold for many kinds of well known fractional integral operators and for various kinds of convex functions.
\end{abstract}

Keywords: Integral operators, fractional integral operators, convex functions, bounds.

MSC: 26D10, 31A10, 26A33.

\section{Introduction}

$\mathbf{T}$ he notion named convexity has applications in almost all branches of mathematics for instance in mathematical analysis, optimization theory, mathematical statistics, graph theory etc. It has been used elegantly for the improvement of solutions of the proposed problems. The fascinating and elegant appearance of convex functions in different forms provides the motivation and encouragement for defining new concepts and definitions. For example the notions of superquadratic function, quasi-convex function, $(\alpha, m)$-convex function, $(h-m)$-convex function, $(s, m)$-convex function, exponentially convex function and many more are due to convex functions, see [1-7] and references therein.

Convex functions are directly related with many known inequalities including Hadamard inequality, Jensen inequality, Arithmetic mean-Geometric mean inequality, Holder inequality etc. Also they have been proved very useful in the establishment of new inequalities. In recent era fractional integral inequalities are in focus of researchers. Many interesting extensions and generalizations for many well known inequalities have been produced by using different types of fractional integrals, see [8-13] and references therein.

The aim of this paper is to establish integral inequalities for $(\alpha, h-m)$-convex functions which have direct consequences to several known fractional integral operators and for functions deducible from $(\alpha, h-$ $m$ )-convex functions. We proceed by giving definitions of generalized fractional integral operators.

Definition 1. [14] Let $f:[a, b] \rightarrow \mathbb{R}$ be an integrable function. Also let $g$ be an increasing and positive function on $(a, b]$, having a continuous derivative $g^{\prime}$ on $(a, b)$. The left-sided and right-sided fractional integrals of a function $f$ with respect to another function $g$ on $[a, b]$ of order $\mu$ where $\Re(\mu)>0$ are defined by

$$
\begin{aligned}
& { }_{g}^{\mu} I_{a^{+}} f(x)=\frac{1}{\Gamma(\mu)} \int_{a}^{x}(g(x)-g(t))^{\mu-1} g^{\prime}(t) f(t) d t, x>a, \\
& { }_{g}^{\mu} I_{b^{-}} f(x)=\frac{1}{\Gamma(\mu)} \int_{x}^{b}(g(t)-g(x))^{\mu-1} g^{\prime}(t) f(t) d t, x<b,
\end{aligned}
$$

where $\Gamma($.$) is the gamma function.$ 
Definition 2. [13] Let $f:[a, b] \rightarrow \mathbb{R}$ be an integrable function. Also let $g$ be an increasing and positive function on $(a, b]$, having a continuous derivative $g^{\prime}$ on $(a, b)$. The left-sided and right-sided fractional integrals of a function $f$ with respect to another function $g$ on $[a, b]$ of order $\mu$ where $\Re(\mu), k>0$ are defined by

$$
\begin{aligned}
& { }_{g}^{\mu} I_{a^{+}}^{k} f(x)=\frac{1}{k \Gamma_{k}(\mu)} \int_{a}^{x}(g(x)-g(t))^{\frac{\mu}{k}-1} g^{\prime}(t) f(t) d t, x>a, \\
& { }_{g}^{\mu} I_{b^{-}}^{k} f(x)=\frac{1}{k \Gamma_{k}(\mu)} \int_{x}^{b}(g(t)-g(x))^{\frac{\mu}{k}-1} g^{\prime}(t) f(t) d t, x<b,
\end{aligned}
$$

where $\Gamma_{k}($.$) is defined as follows$

$$
\Gamma_{k}(x)=\int_{0}^{\infty} t^{x-1} e^{-\frac{t^{k}}{k}} d t, \Re(x)>0
$$

A fractional integral operator containing an extended generalized Mittag-Leffler function in its kernel is defined as follows:

Definition 3. [8] Let $\omega, \mu, \alpha, l, \gamma, c \in \mathbb{C}, \Re(\mu), \Re(\alpha), \Re(l)>0, \Re(c)>\Re(\gamma)>0$ with $p \geq 0, \delta>0$ and $0<k \leq \delta+\Re(\mu)$. Let $f \in L_{1}[a, b]$ and $x \in[a, b]$. Then the generalized fractional integral operators $\epsilon_{\mu, \alpha, l, l, \omega, a^{+}}^{\gamma, \delta} f$ and $\epsilon_{\mu, \alpha, l, \omega, b^{-}}^{\gamma, \delta, c, c} f$ are defined by

$$
\begin{aligned}
& \left(\epsilon_{\mu, \alpha, l, \omega, a^{+}}^{\gamma, \delta, k, c} f\right)(x ; p)=\int_{a}^{x}(x-t)^{\alpha-1} E_{\mu, \alpha, l}^{\gamma, \delta, k, c}\left(\omega(x-t)^{\mu} ; p\right) f(t) d t \\
& \left(\epsilon_{\mu, \alpha, l, \omega, b^{-}}^{\gamma, \delta, k, c} f\right)(x ; p)=\int_{x}^{b}(t-x)^{\alpha-1} E_{\mu, \alpha, l}^{\gamma, \delta, k, c}\left(\omega(t-x)^{\mu} ; p\right) f(t) d t,
\end{aligned}
$$

where

$$
E_{\mu, \alpha, l}^{\gamma, \delta, k, c}(t ; p)=\sum_{n=0}^{\infty} \frac{\beta_{p}(\gamma+n k, c-\gamma)}{\beta(\gamma, c-\gamma)} \frac{(c)_{n k}}{\Gamma(\mu n+\alpha)} \frac{t^{n}}{(l)_{n \delta}}
$$

is the extended generalized Mittag-Leffler function.

The following integral operator unifies all above definitions in a single form.

Definition 4. [15] Let $f, g:[a, b] \longrightarrow \mathbb{R}, 0<a<b$, be the functions such that $f$ be positive and $f \in L_{1}[a, b]$, and $g$ be differentiable and strictly increasing. Also let $\frac{\phi}{x}$ be an increasing function on $[a, \infty)$ and $\alpha, l, \gamma, c \in \mathbb{C}$, $p, \mu, \delta \geq 0$ and $0<k \leq \delta+\mu$. Then for $x \in[a, b]$ the left and right integral operators are defined by:

$$
\begin{aligned}
& \left({ }_{g} F_{\mu, \alpha, \alpha, l, a^{+}}^{\phi, \gamma, \delta, k, c} f\right)(x, \omega ; p)=\int_{a}^{x} K_{x}^{y}\left(E_{\mu, \alpha^{\prime}, l}^{\gamma, \delta, k, c}, g ; \phi\right) f(y) d(g(y)), \\
& \left({ }_{g} F_{\mu, \alpha, \alpha, l, b^{-}}^{\phi, \gamma, \delta, k, c} f\right)(x, \omega ; p)=\int_{x}^{b} K_{y}^{x}\left(E_{\mu, \alpha^{\prime}, l}^{\gamma, \delta, c, c}, g ; \phi\right) f(y) d(g(y)),
\end{aligned}
$$

where the involved kernel is defined by

$$
K_{x}^{y}\left(E_{\mu, \alpha^{\prime}, l}^{\gamma, \delta, k}, g ; \phi\right)=\frac{\phi(g(x)-g(y))}{g(x)-g(y)} E_{\mu, \alpha^{\prime}, l}^{\gamma, \delta, k}\left(\omega(g(x)-g(y))^{\mu} ; p\right) .
$$

For suitable settings of functions $\phi, g$ and certain values of parameters included in Mittag-Leffler function, several recently defined known fractional integrals studied in $[9-11,14,16-25]$ can be reproduced, see [26, Remarks 6 and 7].

Next we give some definitions that generalize the notion of convexity.

First we give the definitions of $(h-m)$-convex and $(\alpha, m)$-convex functions as follows:

Definition 5. [6] Let $J \subseteq \mathbb{R}$ be an interval containing $(0,1)$ and let $h: J \rightarrow \mathbb{R}$ be a non-negative function. A function $f:[0, b] \rightarrow \mathbb{R}$ is called $(h-m)$-convex function, if $f$ is non-negative and for all $x, y \in[0, b], m \in[0,1]$ and $t \in(0,1)$, one has

$$
f(t x+m(1-t) y) \leq h(t) f(x)+m h(1-t) f(y) .
$$


By selecting different values of function $h$ and parameter $m$, the above definition produces many well known definitions.

Remark 1. (i) If $m=1$, then $h$-convex function can be obtained.

(ii) If $h(t)=t$, then $m$-convex function can be obtained.

(iii) If $h(t)=t$ and $m=1$, then convex function can be obtained.

(iv) If $h(t)=1$ and $m=1, p$-function can be obtained.

(v) If $h(t)=t^{s}$ and $m=1$, then $s$-convex function can be obtained.

(vi) If $h(t)=\frac{1}{t}$ and $m=1$, then Godunova-Levin function can be obtained.

(vii) If $h(t)=\frac{1}{t^{s}}$ and $m=1$, then $s$-Godunova-Levin function of second kind can be obtained.

Definition 6. [5] A function $f:[0, b] \rightarrow \mathbb{R}, b>0$ is said to be $(\alpha, m)$-convex, where $(\alpha, m) \in[0,1]^{2}$ if

$$
f(t x+m(1-t) y) \leq t^{\alpha} f(x)+m\left(1-t^{\alpha}\right) f(y)
$$

holds for all $x, y \in[0, b]$ and $t \in[0,1]$.

Remark 2. (i) If we put $(\alpha, m)=(1, m)$, then (13) gives the definition of $m$-convex function.

(ii) If we put $(\alpha, m)=(1,1)$, then (13) gives the definition of convex function.

(iii) If we put $(\alpha, m)=(1,0)$, then (13) gives the definition of star-shaped function.

The next definition unifies the above two definitions elegantly.

Definition 7. [2] Let $J \subseteq \mathbb{R}$ be an interval containing $(0,1)$ and let $h: J \rightarrow \mathbb{R}$ be a non-negative function. A function $f:[0, b] \rightarrow \mathbb{R}$ is called $(\alpha, h-m)$-convex function, if $f$ is non-negative and for all $x, y \in[0, b], t \in(0,1)$ and $(\alpha, m) \in[0,1]^{2}$, one has

$$
f(t x+m(1-t) y) \leq h\left(t^{\alpha}\right) f(x)+m h\left(1-t^{\alpha}\right) f(y) .
$$

One can note that the definitions of $(h-m)$-convex and $(\alpha, m)$-convex functions can be obtained by setting $\alpha=1$ and $h(t)=t$ respectively in (14). Motivated by this generalized convexity we are interested to investigate the bounds of the sum of left and right sided integral operators for these functions. By using $(\alpha, h-m)$-convex functions, upper bounds of (9) and (10) are obtained. Furthermore by using condition of symmetry, two sided Hadamard type bounds are obtained and then by using $(\alpha, h-m)$-convexity of function $\left|f^{\prime}\right|$ and by defining an integral operator for convolution of two functions further bounds are studied.

In [27], we studied the properties of the kernel given in (11). Here we are interested in the following property.

(P) Let $g$ and $\frac{\phi}{I}$ be increasing functions. Then for $m<t<n, m, n \in[a, b]$ the kernel $K_{m}^{n}\left(E_{\mu, \alpha^{\prime}, l}^{\gamma, \delta, c}, g ; \phi\right)$ satisfies the following inequality:

$$
K_{t}^{m}\left(E_{\mu, \alpha^{\prime}, l}^{\gamma, \delta, k, c}, g ; \phi\right) g^{\prime}(t) \leq K_{n}^{m}\left(E_{\mu, \alpha^{\prime}, l}^{\gamma, \delta, k, c}, g ; \phi\right) g^{\prime}(t) .
$$

This can be obtained from following two straightforward inequalities:

$$
\begin{gathered}
\frac{\phi(g(t)-g(m))}{g(t)-g(m)} g^{\prime}(t) \leq \frac{\phi(g(n)-g(m))}{g(n)-g(m)} g^{\prime}(t), \\
E_{\mu, \alpha^{\prime}, l}^{\gamma, \delta, c}\left(\omega(g(t)-g(m))^{\mu} ; p\right) \leq E_{\mu, \alpha^{\prime}, l}^{\gamma, \delta, c}\left(\omega(g(n)-g(m))^{\mu} ; p\right) .
\end{gathered}
$$

The reverse of inequality (15) holds when $g$ and $\frac{\phi}{I}$ are of opposite monotonicity.

\section{Main results}

The following theorem provides upper bound for unified integral operators of $(\alpha, h-m)$-convex functions. 
Theorem 1. Let $f \in L_{1}[a, b]$ be a positive $(\alpha, h-m)$-convex function, $0 \leq a<m b, m \neq 0$. Let $g$ be differentiable and strictly increasing function and $\frac{\phi}{x}$ be an increasing function on $[a, b]$. If $\alpha^{\prime}, \beta, l, \gamma, c \in \mathbb{C}, p, \mu \geq 0, \delta \geq 0$ and $0<k \leq \delta+\mu$, then for $x \in(a, b)$ we have

$$
\begin{aligned}
\left({ }_{g} F_{\mu, \alpha^{\prime}, l, a^{+}}^{\phi, \gamma} f, k, c\right. & (x, \omega ; p)+\left({ }_{g} F_{\mu, \beta, l, b}^{\phi, \gamma, \delta, k, c} f\right)(x, \omega ; p) \\
\leq & K_{x}^{a}\left(E_{\mu, \alpha^{\prime}, l, c}^{\gamma, \delta, k}, \phi\right)(x-a)\left(f(a) H_{x}^{a}\left(z^{\alpha}, h ; g^{\prime}\right)+m f\left(\frac{x}{m}\right) H_{x}^{a}\left(1-z^{\alpha}, h ; g^{\prime}\right)\right) \\
& +K_{b}^{x}\left(E_{\mu, \beta, l}^{\gamma, \delta, k, c}, g ; \phi\right)(b-x)\left(f(b) H_{x}^{b}\left(z^{\alpha}, h ; g^{\prime}\right)+m f\left(\frac{x}{m}\right) H_{x}^{b}\left(1-z^{\alpha}, h ; g^{\prime}\right)\right),
\end{aligned}
$$

where $H_{x}^{a}\left(z^{\alpha}, h ; g^{\prime}\right)=\int_{0}^{1} h\left(z^{\alpha}\right) g^{\prime}(x-z(x-a)) d z$ and $H_{x}^{a}\left(1-z^{\alpha}, h ; g^{\prime}\right)=\int_{0}^{1} h\left(1-z^{\alpha}\right) g^{\prime}(x-z(x-a)) d z$.

Proof. By $(\mathbf{P})$, the following inequalities hold

$$
\begin{aligned}
& K_{x}^{t}\left(E_{\mu, \alpha^{\prime}, l}^{\gamma, \delta, k}, g ; \phi\right) g^{\prime}(t) \leq K_{x}^{a}\left(E_{\mu, \alpha^{\prime}, l}^{\gamma, \delta, k, c}, g ; \phi\right) g^{\prime}(t), a<t<x, \\
& K_{t}^{x}\left(E_{\mu, \alpha^{\prime}, l}^{\gamma, \delta, c}, g ; \phi\right) g^{\prime}(t) \leq K_{b}^{x}\left(E_{\mu, \alpha^{\prime}, l}^{\gamma, \delta, k, c}, g ; \phi\right) g^{\prime}(t), x<t<b .
\end{aligned}
$$

Using $(\alpha, h-m)$-convexity of $f$, we have

$$
\begin{aligned}
& f(t) \leq h\left(\frac{x-t}{x-a}\right)^{\alpha} f(a)+m h\left(1-\left(\frac{x-t}{x-a}\right)^{\alpha}\right) f\left(\frac{x}{m}\right), \\
& f(t) \leq h\left(\frac{t-x}{b-x}\right)^{\alpha} f(b)+m h\left(1-\left(\frac{t-x}{b-x}\right)^{\alpha}\right) f\left(\frac{x}{m}\right) .
\end{aligned}
$$

From (19) and (21), the following integral inequality holds

$$
\begin{aligned}
& \int_{a}^{x} K_{x}^{t}\left(E_{\mu, \alpha^{\prime}, l}^{\gamma, \delta, c}, g ; \phi\right) f(t) d(g(t)) \\
& \quad \leq f(a) K_{x}^{a}\left(E_{\mu, \alpha^{\prime}, l}^{\gamma, \delta, c}, g ; \phi\right) \int_{a}^{x} h\left(\frac{x-t}{x-a}\right)^{\alpha} d(g(t))+m f\left(\frac{x}{m}\right) K_{x}^{a}\left(E_{\mu, \alpha^{\prime}, l}^{\gamma, \delta, c}, g ; \phi\right) \int_{a}^{x} h\left(1-\left(\frac{x-t}{x-a}\right)^{\alpha}\right) d(g(t)) .
\end{aligned}
$$

By using (9) of Definition 4 on left hand side and by setting $z=\frac{x-t}{x-a}$ on right hand side, the following inequality is obtained

$$
\begin{aligned}
& \left({ }_{g} F_{\mu, \alpha^{\prime}, l, a^{+}}^{\phi, \gamma} f\right)(x, \omega ; p) \\
& \leq K_{x}^{a}\left(E_{\mu, \alpha^{\prime}, l}^{\gamma, \delta}, g ; \phi\right)(x-a)\left(f(a) \int_{0}^{1} h\left(z^{\alpha}\right) g^{\prime}(x-z(x-a)) d z+m f\left(\frac{x}{m}\right) \int_{0}^{1} h\left(1-z^{\alpha}\right) g^{\prime}(x-z(x-a)) d z\right) .
\end{aligned}
$$

Above inequality can be written as follows

$$
\left({ }_{g} F_{\mu, \alpha^{\prime}, l, a^{+}}^{\phi, \gamma, \delta} f\right)(x, \omega ; p) \leq K_{x}^{a}\left(E_{\mu, \alpha^{\prime}, l}^{\gamma, \delta, k, c}, g ; \phi\right)(x-a)\left(f(a) H_{x}^{a}\left(z^{\alpha}, h ; g^{\prime}\right)+m f\left(\frac{x}{m}\right) H_{x}^{a}\left(1-z^{\alpha}, h ; g^{\prime}\right)\right) .
$$

On the other hand, multiplying (20) and (22), the following inequality holds

$$
\begin{aligned}
& \int_{x}^{b} K_{t}^{x}\left(E_{\mu, \beta, l}^{\gamma, \delta, k, c}, g ; \phi\right) f(t) d(g(t)) \\
& \quad \leq f(b) K_{b}^{x}\left(E_{\mu, \beta, l}^{\gamma, \delta, k, c}, g ; \phi\right) \int_{x}^{b} h\left(\frac{t-x}{b-x}\right)^{\alpha} d(g(t))+m f\left(\frac{x}{m}\right) K_{b}^{x}\left(E_{\mu, \beta, l}^{\gamma, \delta, k, c}, g ; \phi\right) \int_{x}^{b} h\left(1-\left(\frac{t-x}{b-x}\right)^{\alpha}\right) d(g(t)) .
\end{aligned}
$$

By using (10) of Definition 4 on left hand side and by setting $z=\frac{t-x}{b-x}$ on right hand side, the following inequality is obtained 


$$
\begin{aligned}
\left({ }_{g} F_{\mu, \beta, l, b^{-}}^{\phi, \gamma, \delta, k, c} f\right)(x, \omega ; p) \leq & K_{b}^{x}\left(E_{\mu, \beta, l}^{\gamma, \delta, k, c}, g ; \phi\right)(b-x)\left(f(b) \int_{0}^{1} h\left(z^{\alpha}\right) g^{\prime}(x-z(x-b)) d z\right. \\
& \left.+m f\left(\frac{x}{m}\right) \int_{0}^{1} h\left(1-z^{\alpha}\right) g^{\prime}(x-z(x-b)) d z\right) .
\end{aligned}
$$

Above inequality can be written as follows

$$
\left({ }_{g} F_{\mu, \beta, l, b^{-}}^{\phi, \gamma, \delta, k, c} f\right)(x, \omega ; p) \leq K_{b}^{x}\left(E_{\mu, \beta, l}^{\gamma, \delta, k, c}, g ; \phi\right)(b-x)\left(f(b) H_{x}^{b}\left(z^{\alpha}, h ; g^{\prime}\right)+m f\left(\frac{x}{m}\right) H_{x}^{b}\left(1-z^{\alpha}, h ; g^{\prime}\right)\right) .
$$

By adding (25) and (27), (18) can be obtained.

The following remark summarizes the connection of Theorem 1 with fractional integral inequalities.

Remark 3. (i) If we put $\alpha^{\prime}=\beta$ and $h(t)=t$ in (18), then [28, Theorem 1].

(ii) If we put $\alpha^{\prime}=\beta, m=\alpha=1$ and $h(t)=t$ in (18), then [26, Theorem 8] can be obtained.

(iii) If we put $\phi(t)=\frac{\Gamma\left(\alpha^{\prime}\right) t^{\frac{\alpha^{\prime}}{k}}}{k \Gamma_{k}\left(\alpha^{\prime}\right)}, h(t)=g(t)=t, \alpha=m=1$ and $p=\omega=0$ in (18), then [29, Theorem 1] can be obtained.

(iv) If we put $\alpha^{\prime}=\beta$ in the result of (iii), then [29, Corollary 1] can be obtained.

(v) If we put $k=1$ and $x=a$ or $x=b$ in the result of (iv), then [29, Corollary 2] can be obtained.

(vi) If we put $k=1$ and $x=\frac{a+b}{2}$ in the result of (iv), then [29, Corollary 3] can be obtained.

(vii) If we put $\phi(t)=t^{\alpha^{\prime}}, \alpha=1$ and $g(t)=t$ in (18), then [30, Theorem 1] can be obtained.

(viii) If we put $\phi(t)=t^{\alpha^{\prime}}, \alpha=m=1$ and $h(t)=g(t)=t$ in (18), then [30, Corollary 1] can be obtained.

(ix) If we put $\phi(t)=\Gamma\left(\alpha^{\prime}\right) t^{\alpha^{\prime}}, \alpha=1, p=\omega=0$ and $g(t)=t$ in (18), then [31, Theorem 1] can be obtained.

(x) If we put $\alpha^{\prime}=\beta$ in the result of (ix), then [31, Corollary 1] can be obtained.

(xi) If we put $\alpha^{\prime}=\beta, \phi(t)=\Gamma\left(\alpha^{\prime}\right) t^{\alpha^{\prime}}, p=\omega=0, g(t)=t, \alpha=1$ and using [31, Remark 2] in (18), then [31, Corollary 2] can be obtained.

(xii) If we put $\phi(t)=\Gamma\left(\alpha^{\prime}\right) t^{\alpha^{\prime}}, \alpha=1, p=\omega=0 h(t)=1\left(\right.$ and $\left.h(t)=t^{p}\right)$, respectively and $g(t)=t$ in (18), then [31, Theorem 3] can be obtained.

(xiii) If we put $\phi(t)=\Gamma\left(\alpha^{\prime}\right) t^{\alpha^{\prime}}, p=\omega=0, m=\alpha=1$ and $g(t)=h(t)=t$ in (18), then [32, Theorem 1] can be obtained.

(xiv) If we put $\alpha^{\prime}=\beta$ in the result of (xiii), then [32, Corollary 1] can be obtained.

(xv) If we put $\phi(t)=\Gamma\left(\alpha^{\prime}\right) t^{\alpha^{\prime}}, p=\omega=0, m=\alpha=1$ and $h(t)=t$ in (18), then [33, Theorem 1] can be obtained.

(xvi) If we put $\alpha^{\prime}=\beta, \phi(t)=\Gamma\left(\alpha^{\prime}\right) t^{\alpha^{\prime}}, p=\omega=0, m=\alpha=1$ and $h(t)=t$ in (18), then [33, Corollary 1] can be obtained.

(xvii) If we put $\phi(t)=t^{\alpha^{\prime}}, g(t)=t$ and $h(t)=t^{s}, \alpha=m=1$ in (18), then [34, Theorem 2.1] can be obtained.

(xviii) If we put $\alpha^{\prime}=\beta, \phi(t)=t^{\alpha^{\prime}}, g(t)=t, m=\alpha=1$ and $h(t)=t^{s}$ in (18), then [34, Corollary 2.1] can be obtained.

(xix) If we put $p=\omega=0, \alpha=1$ and $h(t)=t^{s}$ in (18), then [35, Theorem 1] can be obtained.

(xx) If we put $\phi(t)=t^{\alpha^{\prime}}, h(t)=t^{s}, \alpha=1$ and $g(t)=t$ in (18), then [36, Theorem 1] can be obtained.

(xxi) If we put $\alpha^{\prime}=\beta$ in the result of (xx), then [36, Corollary 1] can be obtained.

(xxii) If we put $\phi(t)=\frac{\left.\Gamma\left(\alpha^{\prime}\right) t\right)^{\frac{\alpha^{\prime}}{k}}}{k \Gamma_{k}\left(\alpha^{\prime}\right)}, h(t)=z$ and $p=\omega=0$ in (18), then [12, Theorem 1] can be obtained.

(xxiii) If we put $\alpha^{\prime}=\beta$ in the result of (xxii), then [12, corollary 1] can be obtained.

We have the continuity property for unified integral operators of $(\alpha, h-m)$-convex functions.

Theorem 2. With assumptions of Theorem 1 , if $h \in L_{\infty}[0,1]$ and $f \in L_{\infty}[a, b]$, then unified integral operators for $(h-m)$-convex functions are bounded and continuous.

Proof. From (25) we have

$$
\left|\left({ }_{g} F_{\mu, \alpha^{\prime}, l, a^{+}}^{\phi, \gamma} f\right)(x, \omega ; p)\right| \leq M_{h, K_{b}^{a}}^{\alpha, m}\|f\|_{[a, b]},
$$

where $M_{h, K_{b}^{a}}^{\alpha, m}=K_{b}^{a}\left(E_{\mu, \alpha^{\prime}, l}^{\gamma, \delta, c}, g ; \phi\right)(g(b)-g(a))(m+1)\|h\|_{\infty}$. Similarly, from (27) the following inequality holds

$$
\left|\left({ }_{g} F_{\mu, \beta, l, b^{-}}^{\phi, \gamma, \delta, c} f\right)(x, \omega ; p)\right| \leq M_{h, K_{b}^{a}}^{\alpha, m}\|f\|_{\infty} .
$$


Therefore from linearity and boundedness, the continuity of unified integral operators is followed.

The following lemma is important to proof of the upcoming theorem:

Lemma 1. Let $f:[a, b] \rightarrow \mathbb{R}$, be $(\alpha, h-m)$-convex function, where $0 \leq a<m b, m \neq 0$ and $f(x)=f\left(\frac{a+b-x}{m}\right)$. Then the following inequality holds:

$$
f\left(\frac{a+b}{2}\right) \leq\left(h\left(\frac{1}{2^{\alpha}}\right)+m h\left(\frac{2^{\alpha}-1}{2^{\alpha}}\right)\right) f(x) x \in[a, b] .
$$

Proof. Since $f$ is $(\alpha, h-m)$-convex, therefore the following inequality is valid

$$
\begin{aligned}
f\left(\frac{a+b}{2}\right) & \leq h\left(\frac{1}{2^{\alpha}}\right) f\left(\frac{x-a}{b-a} b+\frac{b-x}{b-a} a\right)+m h\left(\frac{2^{\alpha}-1}{2^{\alpha}}\right) f\left(\frac{\frac{x-a}{b-a} a+\frac{b-x}{b-a} b}{m}\right) \\
& \leq h\left(\frac{1}{2^{\alpha}}\right) f(x)+m h\left(\frac{2^{\alpha}-1}{2^{\alpha}}\right) f\left(\frac{a+b-x}{m}\right) .
\end{aligned}
$$

By using $f(x)=f\left(\frac{a+b-x}{m}\right)$ in above inequality, we get (28).

Remark 4. (i) If we put $h(x)=x$ and $\alpha=m=1$ in (28), then [32, Lemma 1] can be obtained.

(ii) If we put $\alpha=1$ and $h(t)=t^{S}$ in (28), then [35, Lemma 1] can be obtained.

(iii) If we put $h(x)=x$ in (28), then [12, Lemma 1] can be obtained.

(iv) If we put $\alpha=1$ in (28), then [31, Lemma 1] can be obtained.

The following result provides generalized Hadamard inequality for $(\alpha, h-m)$-convex functions.

Theorem 3. Suppose $f, g$ and $\phi$ are as in Theorem 1 and $f(x)=f\left(\frac{a+b-x}{m}\right)$, then we have

$$
\begin{aligned}
& \frac{f\left(\frac{a+b}{2}\right)}{h\left(\frac{1}{2^{\alpha}}\right)+m h\left(\frac{2^{\alpha}-1}{2^{\alpha}}\right)}\left(\left({ }_{g} F_{\mu, \beta, l, l, b^{-}}^{\phi, \gamma, \delta, k} 1\right)(a, \omega ; p)+\left({ }_{g} F_{\mu, \alpha^{\prime}, l, a^{+}}^{\phi, \gamma}, \delta, k, c\right)(b, \omega ; p)\right) \\
& \leq\left({ }_{g} F_{\mu, \alpha^{\prime}, l, a^{+}}^{\phi, \gamma, \delta} f\right)(b, \omega ; p)+\left({ }_{g} F_{\mu,, \beta, l, l, b^{-}}^{\phi, \gamma, c, c} f\right)(a, \omega ; p) \\
& \leq(b-a)\left(K_{b}^{a}\left(E_{\mu, \alpha^{\prime}, l}^{\gamma, \delta, k, c}, g ; \phi\right)+K_{b}^{a}\left(E_{\mu, \beta, l}^{\gamma, \delta, k, c}, g ; \phi\right)\right)\left(f(b) H_{b}^{a}\left(z^{\alpha}, h ; g^{\prime}\right)+m f\left(\frac{a}{m}\right) H_{b}^{a}\left(1-z^{\alpha}, h ; g^{\prime}\right)\right) .
\end{aligned}
$$

Proof. By $(\mathbf{P})$, the following inequalities hold

$$
\begin{aligned}
& K_{x}^{a}\left(E_{\mu, \beta, l}^{\gamma, \delta, k, c}, g ; \phi\right) g^{\prime}(x) \leq K_{b}^{a}\left(E_{\mu, \beta, l}^{\gamma, \delta, k, c}, g ; \phi\right) g^{\prime}(x), a<x<b, \\
& K_{b}^{x}\left(E_{\mu, \alpha^{\prime}, l}^{\gamma, \delta, c}, g ; \phi\right) g^{\prime}(x) \leq K_{b}^{a}\left(E_{\mu, \alpha^{\prime}, l}^{\gamma, \delta, k, c}, g ; \phi\right) g^{\prime}(x) a<x<b .
\end{aligned}
$$

Using $(\alpha, h-m)$-convexity of $f$, we have

$$
f(x) \leq h\left(\frac{x-a}{b-a}\right)^{\alpha} f(b)+m h\left(1-\left(\frac{x-a}{b-a}\right)^{\alpha}\right) f\left(\frac{a}{m}\right) .
$$

Multiplying (30) and (32) and integrating the resulting inequality over $[a, b]$, we obtain

$$
\begin{aligned}
\int_{a}^{b} K_{x}^{a}\left(E_{\mu, \beta, l}^{\gamma, \delta, c, c}, g ; \phi\right) f(x) d(g(x)) \leq & f(b) K_{b}^{a}\left(E_{\mu, \beta, l}^{\gamma, \delta, k, c}, g ; \phi\right) \int_{a}^{b} h\left(\frac{x-a}{b-a}\right)^{\alpha} d(g(x)) \\
& +m f\left(\frac{a}{m}\right) K_{b}^{a}\left(E_{\mu, \beta, l}^{\gamma, \delta, k, c}, g ; \phi\right) \int_{a}^{b} h\left(1-\left(\frac{x-a}{b-a}\right)^{\alpha}\right) d(g(x)) .
\end{aligned}
$$


By using (9) of Definition 4 on left hand side and by supposing $z=\left(\frac{x-a}{b-a}\right)$ on right hand side, the following inequality is obtained

$$
\begin{aligned}
\left({ }_{g} F_{\mu, \beta, l, l, b^{-}}^{\phi, \gamma, \delta} f\right)(a, \omega ; p) \leq & K_{b}^{a}\left(E_{\mu, \alpha^{\prime}, l}^{\gamma, \delta, k, c}, g ; \phi\right)(b-a)\left(f(b) \int_{0}^{1} h\left(z^{\alpha}\right) g^{\prime}(a+z(b-a)) d z\right. \\
& \left.+m f\left(\frac{a}{m}\right) \int_{0}^{1} h\left(1-z^{\alpha}\right) g^{\prime}(a+z(b-a)) d z\right) .
\end{aligned}
$$

Above inequality can be written as

$$
\left({ }_{g} F_{\mu, \beta, l, b^{-}}^{\phi, \gamma, \delta, k, c} f\right)(a, \omega ; p) \leq K_{b}^{a}\left(E_{\mu, \beta, l}^{\gamma, \delta, k, c}, g ; \phi\right)(b-a)\left(f(b) H_{b}^{a}\left(z^{\alpha}, h ; g^{\prime}\right)+m f\left(\frac{a}{m}\right) H_{b}^{a}\left(1-z^{\alpha}, h ; g^{\prime}\right)\right) .
$$

Adopting the same pattern of simplification as we did for (30) and (32), the following inequality can be obtained from (32) and (31)

$$
\left({ }_{g} F_{\mu, \alpha^{\prime}, l, a^{+}}^{\phi, \gamma, \delta, k, c} f\right)(b, \omega ; p) \leq K_{b}^{a}\left(E_{\mu, \alpha^{\prime}, l}^{\gamma, \delta, k, c}, g ; \phi\right)(b-a)\left(f(b) H_{b}^{a}\left(z^{\alpha}, h ; g^{\prime}\right)+m f\left(\frac{a}{m}\right) H_{b}^{a}\left(1-z^{\alpha}, h ; g^{\prime}\right)\right) .
$$

By adding (34) and (35), following inequality can be achieved

$$
\begin{aligned}
& \left({ }_{g} F_{\mu, \alpha^{\prime}, l, a^{+}}^{\phi, \gamma} f\right)(b, \omega ; p)+\left({ }_{g} F_{\mu, \beta, l, l, b^{-}}^{\phi, \gamma} f\right)(a, \omega ; p) \\
& \leq(b-a)\left(K_{b}^{a}\left(E_{\mu, \alpha^{\prime}, l}^{\gamma, \delta, k, c}, g ; \phi\right)+K_{b}^{a}\left(E_{\mu, \beta, l}^{\gamma, \delta, k, c}, g ; \phi\right)\right)\left(f(b) H_{b}^{a}\left(z^{\alpha}, h ; g^{\prime}\right)+m f\left(\frac{a}{m}\right) H_{b}^{a}\left(1-z^{\alpha}, h ; g^{\prime}\right)\right) .
\end{aligned}
$$

Multiplying both sides of (28) by $K_{x}^{a}\left(E_{\mu, \beta, l}^{\gamma, \delta, k, c}, g ; \phi\right) d(g(x))$ and integrating over $[a, b]$, we have

$$
\begin{aligned}
f\left(\frac{a+b}{2}\right) & \int_{a}^{b} K_{x}^{a}\left(E_{\mu, \beta, l}^{\gamma, \delta, k, c}, g ; \phi\right) f(x) d(g(x)) \\
& \leq\left(h\left(\frac{1}{2^{\alpha}}\right)+m h\left(\frac{2^{\alpha}-1}{2^{\alpha}}\right)\right) \int_{a}^{b} K_{x}^{a}\left(E_{\mu, \beta, l}^{\gamma, \delta, k, c}, g ; \phi\right) f(x) d(g(x)) .
\end{aligned}
$$

From Definition 4, the following inequality is obtained

$$
\frac{f\left(\frac{a+b}{2}\right)}{h\left(\frac{1}{2^{\alpha}}\right)+m h\left(\frac{2^{\alpha}-1}{2^{\alpha}}\right)}\left({ }_{g} F_{\mu, \beta, l, b^{-}}^{\phi, \gamma, \delta, k, c} 1\right)(a, \omega ; p) \leq\left({ }_{g} F_{\mu, \beta, l, b^{-}}^{\phi, \gamma, \delta, k, c} f\right)(a, \omega ; p) .
$$

Similarly, multiplying both sides of (28) by $K_{b}^{x}\left(E_{\mu, \alpha^{\prime}}^{\gamma, \delta, k, c}, g ; \phi\right) d(g(x))$ and integrating over $[a, b]$, we have

$$
\frac{f\left(\frac{a+b}{2}\right)}{h\left(\frac{1}{2^{\alpha}}\right)+m h\left(\frac{2^{\alpha}-1}{2^{\alpha}}\right)}\left({ }_{g} F_{\mu, \alpha^{\prime}, l, l, a^{+}}^{\phi, \gamma} 1\right)(b, \omega ; p) \leq\left({ }_{g} F_{\mu, \alpha^{\prime}, l, a^{+}}^{\phi, \gamma} f\right)(b, \omega ; p) .
$$

By adding (37) and (38) following inequality is obtained

$$
\begin{gathered}
\frac{f\left(\frac{a+b}{2}\right)}{h\left(\frac{1}{2^{\alpha}}\right)+m h\left(\frac{2^{\alpha}-1}{2^{\alpha}}\right)}\left(\left({ }_{g} F_{\mu, \beta, l, l, b^{-}}^{\phi, \gamma, \delta} 1\right)(a, \omega ; p)+\left({ }_{g} F_{\mu, \alpha^{\prime}, l, a^{+}}^{\phi, r, \delta, k} 1\right)(b, \omega ; p)\right) \\
\leq\left({ }_{g} F_{\mu, \beta, l, l, b^{-}}^{\phi, \gamma, \delta, k} f\right)(a, \omega ; p)+\left({ }_{g} F_{\mu, \alpha^{\prime}, l, l, a^{+}}^{\phi, \gamma} f\right)(b, \omega ; p) .
\end{gathered}
$$

Using (36) and (39), inequality (29) can be achieved.

The following remark summarizes the connection of Theorem 3 with already known fractional integral inequalities. 
Remark 5. (i) If we put $\alpha^{\prime}=\beta$ and $h(t)=t$ in (29), then [28, Theorem 2].

(ii) If we put $\alpha^{\prime}=\beta, m=\alpha=1$ and $h(t)=t$ in (29), then [26, Theorem 22] can be obtained.

(iii) If we put $\phi(t)=\Gamma\left(\alpha^{\prime}\right) t^{\frac{\alpha^{\prime}}{k}}+1, h(t)=g(t)=t, \alpha=m=1$ and $p=\omega=0$ in (29), then [29, Theorem 3] can be obtained.

(iv) If we put $\alpha^{\prime}=\beta$ in the result of (iii), then [29, Corollary 6] can be obtained.

(v) If we put $\phi(t)=t^{\alpha^{\prime}+1}, \alpha=1$ and $g(t)=t$ in (29), then [30, Theorem 3] can be obtained.

(vi) If we put $m=1$ and $h(t)=t$ in the result of (v), then [30, Corollary 3] can be obtained.

(vii) If we put $\phi(t)=\Gamma\left(\alpha^{\prime}\right) t^{\alpha^{\prime}+1}, \alpha=1, p=\omega=0$ and $g(t)=t$ in (29), then [31, Theorem 3] can be obtained.

(viii) If we put $\alpha^{\prime}=\beta$ in the result of (vii), then [31, Corollary 6] can be obtained.

(ix) If we put $h(t)=t^{p}$ in the result of (viii), then [31, Corollary 7] can be obtained.

(x) If we put $m=1$ in (ix), then [31, Corollary 8] can be obtained.

(xi) If we put $p=1$ in (x), then [31, Corollary 9] can be obtained.

(xii) If we put $\phi(t)=\Gamma\left(\alpha^{\prime}\right) t^{\alpha^{\prime}+1}, p=\omega=0, m=\alpha=1$ and $g(t)=h(t)=t$ in (29), then [32, Theorem 3] can be obtained.

(xiii) If we put $\alpha^{\prime}=\beta, \phi(t)=\Gamma\left(\alpha^{\prime}\right) t^{\alpha^{\prime}}$ in the result of (xii), then [32, Corollary 6] can be obtained.

(xiv) If we put $\phi(t)=\Gamma\left(\alpha^{\prime}\right) t^{\alpha^{\prime}+1}, p=\omega=0, m=\alpha=1$ and $h(t)=t$ in (29), then [33, Theorem 3] can be obtained.

(xv) If we put $\alpha^{\prime}=\beta$ in the result of (xiv), then [33, Corollary 3] can be obtained.

(xvi) If we put $\phi(t)=t^{\alpha^{\prime}+1}, g(t)=t$ and $h(t)=t^{s}, \alpha=m=1$ in (29), then [34, Theorem 2.4] can be obtained.

(xvii) If we put $\alpha^{\prime}=\beta$ in the result of (xvi), then [34, Corollary 2.6] can be obtained.

(xviii) If we put $p=\omega=0, \alpha=1$ and $h(t)=t^{s}$ in (29), then [35, Theorem 3] can be obtained.

(xix) If we put $\phi(t)=\Gamma\left(\alpha^{\prime}\right) t^{\alpha^{\prime}+1}, h(t)=t^{s}, \alpha=1$ and $g(t)=t$ in (29), then [36, Theorem 4] can be obtained.

(xx) If we put $\alpha^{\prime}=\beta$ in the result of (xix), then [36, Corollary 6] can be obtained.

(xxi) If we put $\phi(t)=\Gamma\left(\alpha^{\prime}\right) t^{\frac{\alpha^{\prime}}{k}+1}, h(t)=t$ and $p=\omega=0$ in (29), then [12, Theorem 3] can be obtained.

(xxii) If we put $\alpha^{\prime}=\beta$ in the result of (xxi), then [12, Corollary 3] can be obtained.

The forthcoming result is established for a differentiable function $f$, by utilizing the $(\alpha, h-m)$-convexity of $\left|f^{\prime}\right|$.

Theorem 4. Let $f:[a, b] \longrightarrow \mathbb{R}$ be a differentiable function such that $\left|f^{\prime}\right|$ is $(\alpha, h-m)$-convex $, 0 \leq a<m b, m \neq 0$. Let $g:[a, b] \longrightarrow \mathbb{R}$ be differentiable and strictly increasing function. Also let $\frac{\phi}{x}$ be an increasing function on $[a, b]$. If $\alpha^{\prime}, \beta, l, \gamma, c \in \mathbb{C}, p, \mu \geq 0, \delta \geq 0$ and $0<k \leq \delta+\mu$, then for $x \in(a, b)$ we have

$$
\begin{aligned}
& \left|\left({ }_{g} F_{\mu, \alpha^{\prime}, l, a^{+}}^{\phi, \gamma, \delta, c}(f * g)\right)(x, \omega ; p)+\left({ }_{g} F_{\mu, \beta, l, l, b^{-}}^{\phi, \gamma}(f * g)\right)(x, \omega ; p)\right| \\
& \leq K_{x}^{a}\left(E_{\mu, \alpha^{\prime}, l, l}^{\gamma, \delta, k, c}, \phi\right)(x-a)\left(\left|f^{\prime}(a)\right| H_{x}^{a}\left(z^{\alpha}, h ; g^{\prime}\right)+m\left|f^{\prime}\left(\frac{x}{m}\right)\right| H_{x}^{a}\left(1-z^{\alpha}, h ; g^{\prime}\right)\right) \\
& \quad+K_{b}^{x}\left(E_{\mu, \beta, l, l}^{\gamma, \delta, k, c}, g ; \phi\right)(b-x)\left(\left|f^{\prime}(b)\right| H_{x}^{b}\left(z^{\alpha}, h ; g^{\prime}\right)+m\left|f^{\prime}\left(\frac{x}{m}\right)\right| H_{x}^{b}\left(1-z^{\alpha}, h ; g^{\prime}\right)\right),
\end{aligned}
$$

where

$$
\left({ }_{g} F_{\mu, \alpha^{\prime}, l, a^{+}}^{\phi, \gamma, \delta} f * g\right)(x, \omega ; p):=\int_{a}^{x} K_{x}^{t}\left(E_{\mu, \alpha^{\prime}, l}^{\gamma, \delta, k}, g ; \phi\right) f^{\prime}(t) d(g(t))
$$

and

$$
\left({ }_{g} F_{\mu, \beta, l, b b^{-}}^{\phi, \gamma, \delta} f * g\right)(x, \omega ; p):=\int_{x}^{b} K_{t}^{x}\left(E_{\mu, \alpha^{\prime}, l}^{\gamma, \delta, k, c}, g ; \phi\right) f^{\prime}(t) d(g(t)) .
$$

Proof. Using $(\alpha, h-m)$-convexity of $\left|f^{\prime}\right|$, we have

$$
\left|f^{\prime}(t)\right| \leq h\left(\frac{x-t}{x-a}\right)^{\alpha}\left|f^{\prime}(a)\right|+m h\left(1-\left(\frac{x-t}{x-a}\right)^{\alpha}\right)\left|f^{\prime}\left(\frac{x}{m}\right)\right| .
$$

Now, (41) can be written as follows

$$
\begin{aligned}
& -\left(h\left(\frac{x-t}{x-a}\right)^{\alpha}\left|f^{\prime}(a)\right|+m h\left(1-\left(\frac{x-t}{x-a}\right)^{\alpha}\right)\left|f^{\prime}\left(\frac{x}{m}\right)\right|\right) \\
& \leq f^{\prime}(t) \leq\left(h\left(\frac{x-t}{x-a}\right)^{\alpha}\left|f^{\prime}(a)\right|+m h\left(1-\left(\frac{x-t}{x-a}\right)^{\alpha}\right)\left|f^{\prime}\left(\frac{x}{m}\right)\right|\right) .
\end{aligned}
$$

Let we consider the right hand side inequality of (42) 


$$
f^{\prime}(t) \leq\left(h\left(\frac{x-t}{x-a}\right)^{\alpha}\left|f^{\prime}(a)\right|+m h\left(1-\left(\frac{x-t}{x-a}\right)^{\alpha}\right)\left|f^{\prime}\left(\frac{x}{m}\right)\right|\right)
$$

Multiplying (19) and (43) and integrating over $[a, x]$, we obtain

$$
\begin{aligned}
& \int_{a}^{x} K_{x}^{t}\left(E_{\mu, \alpha^{\prime}, l}^{\gamma, \delta, k, c}, g ; \phi\right) f^{\prime}(t) d(g(t)) \\
& \leq\left|f^{\prime}(a)\right| K_{x}^{a}\left(E_{\mu, \alpha^{\prime}, l}^{\gamma, \delta, k, c}, g\right) \int_{a}^{x} h\left(\frac{x-t}{x-a}\right)^{\alpha} d(g(t))+m\left|f^{\prime}\left(\frac{x}{m}\right)\right| K_{x}^{a}\left(E_{\mu, \alpha^{\prime}, l}^{\gamma, \delta, k, c}, \phi\right) \int_{a}^{x} h\left(1-\left(\frac{x-t}{x-a}\right)^{\alpha}\right) d(g(t)) .
\end{aligned}
$$

By using (9) of Definition 4 on left hand side and by supposing $z=\left(\frac{x-t}{x-a}\right)$ on right hand side, the following inequality is obtained

$$
\begin{aligned}
\left({ }_{g} F_{\mu, \alpha^{\prime}, l, a^{+}}^{\phi, \gamma, \delta, k, c} f\right)(x, \omega ; p) \leq & K_{x}^{a}\left(E_{\mu, \alpha^{\prime}, l}^{\gamma, \delta, k, c}, g ; \phi\right)(x-a)\left(\left|f^{\prime}(a)\right| \int_{0}^{1} h\left(z^{\alpha}\right) g^{\prime}(x-z(x-a)) d z\right. \\
& \left.+m\left|f^{\prime}\left(\frac{x}{m}\right)\right| \int_{0}^{1} h\left(1-z^{\alpha}\right) g^{\prime}(x-z(x-a)) d z\right) .
\end{aligned}
$$

Above inequality can be written as

$$
\left({ }_{g} F_{\mu, \alpha^{\prime}, l, a^{+}}^{\phi, \gamma, \delta, k, c} f\right)(x, \omega ; p) \leq K_{x}^{a}\left(E_{\mu, \alpha^{\prime}, l}^{\gamma, \delta, k, c}, \phi\right)(x-a)\left(\left|f^{\prime}(a)\right| H_{x}^{a}\left(z^{\alpha}, h ; g^{\prime}\right)+m\left|f^{\prime}\left(\frac{x}{m}\right)\right| H_{x}^{a}\left(1-z^{\alpha}, h ; g^{\prime}\right)\right)
$$

If we consider the left hand side from the inequality (42) and adopting the same pattern as we did for the right hand side inequality, we have

$$
\left({ }_{g} F_{\mu, \alpha^{\prime}, l, a^{+}}^{\phi, \gamma, c, k} f\right)(x, \omega ; p) \geq-K_{x}^{a}\left(E_{\mu, \alpha^{\prime}, l}^{\gamma, \delta, k, c}, g ; \phi\right)(x-a)\left(\left|f^{\prime}(a)\right| H_{x}^{a}\left(z^{\alpha}, h ; g^{\prime}\right)+m\left|f^{\prime}\left(\frac{x}{m}\right)\right| H_{x}^{a}\left(1-z^{\alpha}, h ; g^{\prime}\right)\right) .
$$

From (44) and (45), following inequality is observed

$$
\left|\left({ }_{g} F_{\mu, \alpha^{\prime}, l, a^{+}}^{\phi, \gamma, \delta, k} f\right)(x, \omega ; p)\right| \leq K_{x}^{a}\left(E_{\mu, \alpha^{\prime}, l}^{\gamma, \delta, k, c}, g ; \phi\right)(x-a)\left(\left|f^{\prime}(a)\right| H_{x}^{a}\left(z^{\alpha}, h ; g^{\prime}\right)+m\left|f^{\prime}\left(\frac{x}{m}\right)\right| H_{x}^{a}\left(1-z^{\alpha}, h ; g^{\prime}\right)\right) \text {. }
$$

Now using $(\alpha, h-m)$-convexity of $\left|f^{\prime}\right|$, we have

$$
\left|f^{\prime}(t)\right| \leq h\left(\frac{t-x}{b-x}\right)^{\alpha}\left|f^{\prime}(b)\right|+m h\left(1-\left(\frac{t-x}{b-x}\right)^{\alpha}\right)\left|f^{\prime}\left(\frac{x}{m}\right)\right|
$$

On the same pattern as we did for (19) and (41), one can get following inequality from (20) and (47)

$$
\begin{aligned}
\left|\left({ }_{g} F_{\mu, \beta, l, b^{-}}^{\phi, \gamma, \delta, k, c} f\right)(x, \omega ; p)\right| \leq & K_{b}^{x}\left(E_{\mu, \beta, l}^{\gamma, \delta, k, c}, g ; \phi\right)(b-x)\left(\left|f^{\prime}(b)\right| \int_{0}^{1} h\left(z^{\alpha}\right) g^{\prime}(x-z(x-b)) d z\right. \\
& \left.+m\left|f^{\prime}\left(\frac{x}{m}\right)\right| \int_{0}^{1} h\left(1-z^{\alpha}\right) g^{\prime}(x-z(x-b)) d z\right) .
\end{aligned}
$$

Above inequality can be written as

$$
\left|\left({ }_{g} F_{\mu, \beta, l, b^{-}}^{\phi, \gamma, \delta, c} f\right)(x, \omega ; p)\right| \leq K_{b}^{x}\left(E_{\mu, \beta, l}^{\gamma, \delta, k, c}, g ; \phi\right)(b-x)\left(\left|f^{\prime}(b)\right| H_{x}^{b}\left(z^{\alpha}, h ; g^{\prime}\right)+m\left|f^{\prime}\left(\frac{x}{m}\right)\right| H_{x}^{b}\left(1-z^{\alpha}, h ; g^{\prime}\right)\right) .
$$

By adding (46) and (48), inequality (40) can be achieved.

The following remark summarizes the connection of Theorem 4 with already known results.

Remark 6. (i) If we put $\alpha^{\prime}=\beta$ and $h(t)=t$ in (40), then [28, Theorem 3].

(ii) If we put $\alpha^{\prime}=\beta, m=\alpha=1$ and $h(t)=t$ in (40), then [26, Theorem 25] can be obtained.

(iii) If we put $\phi(t)=\Gamma\left(\alpha^{\prime}\right) t^{\frac{\alpha^{\prime}}{k}+1}, h(t)=g(t)=t, \alpha=m=1$ and $p=\omega=0$ in (40), then [29, Theorem 2] can be obtained.

(iv) If we put $\alpha^{\prime}=\beta$ in the result of (iii), then [29, Corollary 4] can be obtained.

(v) If we put $\phi(t)=t^{\alpha^{\prime}}, \alpha=1$ and $g(t)=t$ in (40), then [30, Theorem 2] can be obtained. 
(vi) If we put $m=1$ and $h(t)=t$ in the result of $(\mathrm{v})$, then [30, Corollary 2] can be obtained.

(vii) If we put $\phi(t)=\Gamma\left(\alpha^{\prime}\right) t^{\alpha^{\prime}+1}, \alpha=1, p=\omega=0$ and $g(t)=t$ in (40), then [31, Theorem 2] can be obtained.

(viii) If we put $\alpha^{\prime}=\beta$ in the result of (vii), then [31, Corollary 4] can be obtained.

(ix) If we put $\phi(t)=\Gamma\left(\alpha^{\prime}\right) t^{\alpha^{\prime}+1}, p=\omega=0, m=\alpha=1$ and $g(t)=h(t)=t$ in (40), then [32, Theorem 2] can be obtained.

(x) If we put $\alpha^{\prime}=\beta, \phi(t)=\Gamma\left(\alpha^{\prime}\right) t^{\alpha^{\prime}}$ in the result of (ix), then [32, Corollary 4] can be obtained.

(xi) If we put $\phi(t)=\Gamma\left(\alpha^{\prime}\right) t^{\alpha^{\prime}+1}, p=\omega=0, m=\alpha=1$ and $h(t)=t$ in (40), then [33, Theorem 2] can be obtained.

(xii) If we put $\alpha^{\prime}=\beta$ in the result of (xi), then [33, Corollary 2] can be obtained.

(xiii) If we put $\phi(t)=t^{\alpha^{\prime} 1}, g(t)=t$ and $h(t)=t^{s}, \alpha=m=1$ in (40), then [34, Theorem 2.3] can be obtained.

(xiv) If we put $\alpha^{\prime}=\beta$ in the result of (xiii), then [34, Corollary 2.5] can be obtained.

(xv) If we put $p=\omega=0, \alpha=1$ and $h(t)=t^{s}$ in (40), then [35, Theorem 2] can be obtained.

(xvi) If we put $\phi(t)=\Gamma\left(\alpha^{\prime}\right) t^{\alpha^{\prime}}, h(t)=t^{s}, \alpha=1$ and $g(t)=t$ in (40), then [36, Theorem 3] can be obtained.

(xvii) If we put $\alpha^{\prime}=\beta$ in the result of (xvi), then [36, Corollary 5] can be obtained.

(xviii) If we put $\phi(t)=\Gamma\left(\alpha^{\prime}\right) t^{\frac{\alpha^{\prime}}{k}+1}, h(t)=t$ and $p=\omega=0$ in (40), then [12, Theorem 2] can be obtained.

(xix) If we put $\alpha^{\prime}=\beta$ in the result of (xviii), then [12, Corollary 2] can be obtained.

In the upcoming section, we will prove the results for $(h-m)$-convex functions. Methodology to prove these results will be same as we use in our main section.

\section{Results for $(h-m)$-convex functions}

In this section we give results for $(h-m)$-convex functions, that are deduced from main results.

Theorem 5. If $h \in L_{\infty}[0,1]$, then for $(h-m)$-convex function $f$, the following inequality holds

$$
\begin{aligned}
\left({ }_{g} F_{\mu, \alpha^{\prime}, l, a^{+}}^{\phi, \gamma, \delta, k, c} f\right) & (x, \omega ; p)+\left({ }_{g} F_{\mu, \beta, l, b^{-}}^{\phi, \gamma, \delta, k, c} f\right)(x, \omega ; p) \\
\leq & \left((g(x)-g(a)) K_{x}^{a}\left(E_{\mu, \alpha^{\prime}, l, g}^{\gamma, \delta, k, c}, \phi\right)\left(m f\left(\frac{x}{m}\right)+f(a)\right)\right. \\
& \left.+(g(b)-g(x)) K_{b}^{x}\left(E_{\mu, \beta, l}^{\gamma, \delta, k, c}, g ; \phi\right)\left(f(b)+m f\left(\frac{x}{m}\right)\right)\right)\|h\|_{\infty} .
\end{aligned}
$$

Proof. By putting $\alpha=1$ in (24), we get

$$
\begin{aligned}
& \left({ }_{g} F_{\mu, \alpha^{\prime}, l, a^{+}}^{\phi, \gamma, \delta, k, c} f\right)(x, \omega ; p) \\
& \quad \leq K_{x}^{a}\left(E_{\mu, \alpha^{\prime}, l}^{\gamma, \delta, k, c}, g ; \phi\right)(x-a)\left(f(a) \int_{0}^{1} g^{\prime}(x-z(x-a)) d z+m f\left(\frac{x}{m}\right) \int_{0}^{1} g^{\prime}(z(x-a)+a) d z\right)\|h\|_{\infty}
\end{aligned}
$$

which further simplifies as follows

$$
\left({ }_{g} F_{\mu, \alpha^{\prime}, l, a^{+}}^{\phi, \gamma, \delta, k} f\right)(x, \omega ; p) \leq(g(x)-g(a)) K_{x}^{a}\left(E_{\mu, \alpha^{\prime}, l}^{\gamma, \delta, k, c}, g ; \phi\right)\left(m f\left(\frac{x}{m}\right)+f(a)\right)\|h\|_{\infty} .
$$

Similarly, by putting $\alpha=1$ in (26) and then computing integral, we get

$$
\left({ }_{g} F_{\mu, \beta, l, b^{-}}^{\phi, \gamma, \delta, k} f\right)(x, \omega ; p) \leq(g(b)-g(x)) K_{b}^{x}\left(E_{\mu, \beta, l}^{\gamma, \delta, k, c}, g ; \phi\right)\left(f(b)+m f\left(\frac{x}{m}\right)\right)\|h\|_{\infty} .
$$

From (50) and (51), the bound in (49) can be achieved.

In the following theorem, we establish Hadamard type inequality for $(h-m)$-convex functions:

Theorem 6. The conditions on $f, g$ and $\phi$ are same as in Theorem 5 and in addition if $f(x)=f\left(\frac{a+b-x}{m}\right)$, then we have

$$
\begin{gathered}
\frac{f\left(\frac{a+b}{2}\right)}{(m+1) h\left(\frac{1}{2}\right)}\left(\left({ }_{g} F_{\mu, \beta, l, b^{-}}^{\phi, \gamma, \delta, k, c} 1\right)(a, \omega ; p)+\left({ }_{g} F_{\mu, \alpha^{\prime}, l, a^{+}}^{\phi, \gamma, \delta, k, c} 1\right)(b, \omega ; p)\right) \leq\left({ }_{g} F_{\mu, \beta, l, b^{-}}^{\phi, \gamma, \delta, k, c} f\right)(a, \omega ; p)+\left({ }_{g} F_{\mu, \alpha^{\prime}, l, a^{+}}^{\phi, \gamma, \delta, k, c} f\right)(b, \omega ; p) \\
\leq(g(b)-g(a))\left(K_{b}^{a}\left(E_{\mu, \alpha^{\prime}, l}^{\gamma, \delta, k, c}, g ; \phi\right)+K_{b}^{a}\left(E_{\mu, \beta, l}^{\gamma, \delta, k, c}, g ; \phi\right)\right)\left(m f\left(\frac{b}{m}\right)+f(a)\right)\|h\|_{\infty}
\end{gathered}
$$


Proof. By putting $\alpha=1$ in (36) and then computing integral, we get

$$
\begin{aligned}
\left({ }_{g} F_{\mu, a^{\prime}, l, a^{+}}^{\phi, \gamma, \delta, k, c} f\right) & (b ; p)+\left({ }_{g} F_{\mu, \beta, l, b^{-}}^{\phi, \gamma, \delta, k, c} f\right)(a ; p) \\
& \leq(g(b)-g(a))\left(K_{b}^{a}\left(E_{\mu, \alpha^{\prime}, l}^{\gamma, \delta, c}, g ; \phi\right)+K_{b}^{a}\left(E_{\mu, \beta, l}^{\gamma, \delta, k, c}, g ; \phi\right)\right)\left(m f\left(\frac{b}{m}\right)+f(a)\right)\|h\|_{\infty} .
\end{aligned}
$$

Similarly, putting $\alpha=1$ in (39), we get

$$
\begin{gathered}
\frac{f\left(\frac{a+b}{2}\right)}{(m+1) h\left(\frac{1}{2}\right)}\left(\left({ }_{g} F_{\mu, \beta, l, b^{-}}^{\phi, \gamma, \delta, k, c} 1\right)(a, \omega ; p)+\left({ }_{g} F_{\mu, \alpha^{\prime}, l, a^{+}}^{\phi, \gamma, \delta, k, c} 1\right)(b, \omega ; p)\right) \\
\leq\left({ }_{g} F_{\mu, \beta, l, l, b^{-}}^{\phi, \gamma, \delta, k, c}\right)(a, \omega ; p)+\left({ }_{g} F_{\mu, \alpha^{\prime}, l, a^{+}}^{\phi, \gamma} f\right)(b, \omega ; p) .
\end{gathered}
$$

From inequalities (53) and (54), inequality (52) can be obtained.

Theorem 7. If $h \in L_{\infty}[0,1]$, then for $(h-m)$-convex function $\left|f^{\prime}\right|$, the following inequality holds

$$
\begin{aligned}
& \left|\left({ }_{g} F_{\mu, \alpha^{\prime}, l, a^{+}}^{\phi, \gamma, \delta, k, c}(f * g)\right)(x, \omega ; p)+\left({ }_{g} F_{\mu, \beta, l, l, b^{-}}^{\phi, \gamma, \delta, c}(f * g)\right)(x, \omega ; p)\right| \\
& \leq\left((g(x)-g(a)) K_{x}^{a}\left(E_{\mu, \alpha^{\prime}, l}^{\gamma, \delta, c, c}, g ; \phi\right)\left(m\left|f^{\prime}\left(\frac{x}{m}\right)\right|+\left|f^{\prime}(a)\right|\right)\right. \\
& \left.+(g(b)-g(x)) K_{b}^{x}\left(E_{\mu, \beta, l}^{\gamma, \delta, k, c}, g ; \phi\right)\left(\left|f^{\prime}(b)\right|+m\left|f^{\prime}\left(\frac{x}{m}\right)\right|\right)\right)\|h\|_{\infty,}
\end{aligned}
$$

Proof. By putting $\alpha=1$ in (46), and then computing integral, we get

$$
\left|\left(g_{\mu} F_{\mu, \alpha^{\prime}, l, a^{+}}^{\phi, \gamma, k, c}(f * g)\right)(x, \omega ; p)\right| \leq(g(x)-g(a)) K_{x}^{a}\left(E_{\mu, \alpha^{\prime}, l}^{\gamma, \delta, c}, g ; \phi\right)\left(m\left|f^{\prime}\left(\frac{x}{m}\right)\right|+\left|f^{\prime}(a)\right|\right)\|h\|_{\infty} .
$$

By putting $\alpha=1$ in (48), and then computing integral, we get

$$
\left|\left({ }_{g} F_{\mu, \beta, l, b^{-}}^{\phi, \gamma, \delta, k, c}(f * g)\right)(x, \omega ; p)\right| \leq(g(b)-g(x)) K_{b}^{x}\left(E_{\mu, \alpha^{\prime}, l}^{\gamma, \delta, c}, g ; \phi\right)\left(\left|f^{\prime}(b)\right|+m\left|f^{\prime}\left(\frac{x}{m}\right)\right|\right)\|h\|_{\infty} .
$$

By adding inequalities (56) and (57), inequality (55) can be achieved.

\section{Some applications to fractional inequalities}

In this section, by applying main results we give some interesting consequences.

\subsection{Hadamard inequalities}

By applying Theorem 3 we give Hadamard inequalities for (9) and (10) for $(\alpha, h-m)$-convex functions.

Corollary 8. If we put $\phi(t)=\frac{\Gamma\left(\alpha^{\prime}\right) t^{\frac{\alpha^{\prime}}{k}}}{k \Gamma_{k}\left(\alpha^{\prime}\right)}$ and $p=\omega=0$ for $\alpha^{\prime}=\beta$ in (29), then the Hadamard inequality for fractional integral operators defined in [13] of $(\alpha, h-m)$-convex functions is obtained as follows

$$
\begin{aligned}
& \frac{2 f\left(\frac{a+b}{2}\right)(g(b)-g(a))^{\alpha^{\prime} / k}}{k \Gamma_{k}\left(\alpha^{\prime}\right)\left(h\left(\frac{1}{2^{\alpha}}\right)+m h\left(\frac{2^{\alpha}-1}{2^{\alpha}}\right)\right)} \leq{ }_{g}^{\alpha^{\prime}} I_{b_{-}}^{k} f(a)+{ }_{g}^{\alpha^{\prime}} I_{a^{+}}^{k} f(b) \\
& \quad \leq \frac{2(b-a)(g(b)-g(a))^{\frac{\alpha^{\prime}}{k}-1}}{k \Gamma_{k}\left(\alpha^{\prime}\right)}\left(f(a) H_{b}^{a}\left(z^{\alpha}, h ; g^{\prime}\right)+m f\left(\frac{b}{m}\right) H_{b}^{a}\left(1-z^{\alpha}, h ; g^{\prime}\right)\right), \quad \alpha^{\prime} \geq k .
\end{aligned}
$$

Corollary 9. If we put $k=1$ in Corollary 8, then the Hadamard inequality for fractional integral operators defined in [14] of $(\alpha, h-m)$-convex functions is obtained as follows 


$$
\begin{gathered}
\frac{2 f\left(\frac{a+b}{2}\right)(g(b)-g(a))^{\alpha^{\prime}}}{\Gamma\left(\alpha^{\prime}\right)\left(h\left(\frac{1}{2^{\alpha}}\right)+m h\left(\frac{2^{\alpha}-1}{2^{\alpha}}\right)\right)} \leq{ }_{g}^{\alpha^{\prime}} I_{b_{-}} f(a)+{ }_{g}^{\alpha^{\prime}} I_{a^{+}} f(b) \\
\quad \leq \frac{2(b-a)(g(b)-g(a))^{\alpha^{\prime}-1}}{\Gamma\left(\alpha^{\prime}\right)}\left(f(a) H_{b}^{a}\left(z^{\alpha}, h ; g^{\prime}\right)+m f\left(\frac{b}{m}\right) H_{b}^{a}\left(1-z^{\alpha}, h ; g^{\prime}\right)\right) .
\end{gathered}
$$

Corollary 10. If we put $g(x)=x$ in Corollary 8 , then the Hadamard inequality for fractional integral operators defined in [19] of $(\alpha, h-m)$-convex functions is obtained as follows

$$
\begin{aligned}
& \frac{2 f\left(\frac{a+b}{2}\right)(b-a)^{\alpha^{\prime} / k}}{\left(h\left(\frac{1}{2^{\alpha}}\right)+m h\left(\frac{2^{\alpha}-1}{2^{\alpha}}\right)\right) k \Gamma_{k}\left(\alpha^{\prime}\right)} \leq{ }^{\alpha^{\prime}} I_{b_{-}}^{k} f(a)+{ }^{\alpha^{\prime}} I_{a^{+}}^{k} f(b) \\
& \quad \leq \frac{2(b-a)^{\frac{\alpha^{\prime}}{k}+1}}{k \Gamma_{k}\left(\alpha^{\prime}\right)}\left(f(a) \int_{0}^{1} h\left(z^{\alpha}\right) d z+m f\left(\frac{b}{m}\right) \int_{0}^{1} h\left(1-z^{\alpha}\right) d z\right), \quad \alpha^{\prime} \geq k .
\end{aligned}
$$

Corollary 11. If we put $g(x)=x$ in Corollary 9, then the Hadamard inequality for fractional integral operators defined in [14] of $(\alpha, h-m)$-convex functions is obtained as follows

$$
\begin{gathered}
\frac{2 f\left(\frac{a+b}{2}\right)(b-a)^{\alpha^{\prime}}}{\left(h\left(\frac{1}{2^{\alpha}}\right)+m h\left(\frac{2^{\alpha}-1}{2^{\alpha}}\right)\right) \Gamma\left(\alpha^{\prime}\right)} \leq{ }^{\alpha^{\prime}} I_{b_{-}} f(a)+{ }^{\alpha^{\prime}} I_{a^{+}} f(b) \\
\leq \frac{2(b-a)^{\alpha^{\prime}+1}}{\Gamma\left(\alpha^{\prime}\right)}\left(f(a) \int_{0}^{1} h\left(z^{\alpha}\right) d z+m f\left(\frac{b}{m}\right) \int_{0}^{1} h\left(1-z^{\alpha}\right) d z\right) .
\end{gathered}
$$

Remark 7. The applications of other theorems in main results are similar to the applications of Theorem 1 and left for the reader.

\section{Concluding Remarks}

This paper provides a detail study of a unified integral operator by using a generalized notion namely $(\alpha, h-m)$-convex function. The presented results are generalized which give the bounds of various well known and independently defined integral operators simultaneously. These results also hold for convex, $m$-convex, $h$-convex, $(s, m)$-convex, $(h-m)$-convex, $(\alpha, m)$-convex functions.

Acknowledgments: We thank to the editor and referees for their careful reading and valuable suggestions to make the article friendly readable.

Author Contributions: All authors contributed equally to the writing of this paper. All authors read and approved the final manuscript.

Conflicts of Interest: "The authors declare no conflict of interest".

\section{References}

[1] Abramovich, S., Jameson, G., \& Sinnamon, G. (2004). Refining Jensen's inequality. Bulletin mathématique de la Société des Sciences Mathématiques de Roumanie, 47(1-2), 3-14.

[2] Farid, G., Rehman, A. U., \& Ain, Q. U. (2020). K-fractional integral inequalities of Hadamard type for $(h-m)-$ convex functions. Computational Methods for Differential Equations, 8(1), 119-140.

[3] Ion, D. A. (2007). Some estimates on the Hermite-Hadamard inequality through quasi-convex functions. Annals of the University of Craiova-Mathematics and Computer Science Series, 34, 82-87.

[4] Quang, X., Farid, G., Pečarić, J., \& Akbar, S. B. (2020). Generalized fractional integral inequalities for exponentially $(s, m)$-convex functions. Journal of Inequalities and Appllication, 2020, Article No 70.

[5] Mihesan, V. G. (1993). A Generalization of the Convexity. Seminar on Functional Equations, Approx. and Convex, Cluj-Napoca, Romania.

[6] Özdemir, M. E., Akdemri, A. O., \& Set, E. (2016). On $(h-m)$-convexity and Hadamard-type inequalities. Transylvanian Journal of Mathematics and Mechanics, 8(1), 51-58. 
[7] Varošanec, S. (2007). On h-convexity. Journal of Mathematical Analysis and Applications, 326(1), 303-311.

[8] Andrić, M., Farid, G., \& Pečarić, J. (2018). A further extension of Mittag-Leffler function. Fractional Calculus and Applied Analysis, 21(5), 1377-1395.

[9] Chen, H., \& Katugampola, U. N. (2017). Hermite-Hadamard and Hermite-Hadamard-Fejér type inequalities for generalized fractional integrals. Journal of Mathematical Analysis and Applications, 446, 1274-1291.

[10] Dragomir, S. S. (2018). Inequalities of Jensens type for generalized $k$-g-fractional integrals of functions for which the composite $f \circ g^{-1}$ is convex. Fractional Differential Calculus, 8(1), 127-150.

[11] Habib, S., Mubeen, S., \& Naeem, M. N. (2018). Chebyshev type integral inequalities for generalized $k$-fractional conformable integrals. Journal of Inequalities and Special Functions, 9(4), 53-65.

[12] Kang, S. M., Farid, G., Waseem, M., Ullah, S., Nazeer, W., \& Mehmood, S. (2019). Generalized $k$-fractional integral inequalities associated with $(\alpha, m)$-convex functions. Journal of Inequalties and Applications, 2019, Article No 255.

[13] Kwun, Y. C., Farid, G., Nazeer, W., Ullah, S., \& Kang, S. M. (2018). Generalized riemann-liouville $k$-fractional integrals associated with Ostrowski type inequalities and error bounds of hadamard inequalities. IEEE access, 6, 64946-64953.

[14] Kilbas, A. A., Srivastava, H. M., \& Trujillo, J. J. (2006). Theory and Applications of Fractional Differential Equations (Vol. 204). North-Holland Mathematics Studies, 204, Elsevier, New York-London.

[15] Farid, G. (2020). A unified integral operator and further its consequences. Open Journal of Mathematical Analysis, 4(1), 1-7.

[16] Farid, G. (2019). Existence of an integral operator and its consequences in fractional and conformable integrals. Open Journal of Mathematical Sciences, 3(3), 210-216.

[17] Jarad, F., Ugurlu, E., Abdeljawad, T., \& Baleanu, D. (2017). On a new class of fractional operators. Advances in Difference Equations, 2017, Article No 247.

[18] Khan, T. U., \& Khan, M. A. (2019). Generalized conformable fractional operators. Journal of Computational and Applied Mathematics, 346, 378-389.

[19] Mubeen, S., \& Habibullah, G. M. (2012). k-Fractional integrals and applications. International Journal of Contemporary Mathematical Sciences, 7(2), 89-94.

[20] Prabhakar, T. R. (1971). A singular integral equation with a generalized Mittag Leffler function in the kernel. Yokohama Mathematical Journal, 19, 7-15.

[21] Rahman, G., Baleanu, D., Qurashi, M. A., Purohit, S. D., Mubeen, S., \& Arshad, M. (2017). The extended Mittag-Leffler function via fractional calculus. Journal of Nonlinear Sciences and Applications, 10(8), 4244-4253.

[22] Salim, T. O., \& Faraj, A. W. (2012). A generalization of Mittag-Leffler function and integral operator associated with fractional calculus. Fractional Calculus and Applied Analysis, 3(5), 1-13.

[23] Sarikaya, M. Z., Dahmani, Z., KIRIS, M. E., \& Ahmad, F. (2016). (k,s)-Riemann-Liouville fractional integral and applications. Hacettepe Journal of Mathematics and Statistics, 45(1), 77-89.

[24] Srivastava, H. M., \& Tomovski, Ž. (2009). Fractional calculus with an integral operator containing a generalized Mittag-Leffler function in the kernel. Applied Mathematics and Computation, 211(1), 198-210.

[25] Tuba, T., Budak, H., Fuat, ., \& Sarikaya, M. Z. (2017). On new generalized fractional integral operators and related fractional inequalities. Konuralp Journal of Mathematics, 8(2), 268-278.

[26] Kwun, Y. C., Farid, G., Ullah, S., Nazeer, W., Mahreen, K., \& Kang, S. M. (2019). Inequalities for a unified integral operator and associated results in fractional calculus. IEEE Access, 7, 126283-126292.

[27] He, Z., Ma, X., Farid, G., Haq, A. U., \& Mahreen, K. (2020). Bounds of a unified integral operator for $(s, m)-$ convex functions and their consequences. AIMS Mathematics, 5(6), 5510-5520.

[28] Farid, G., Ni, B., \& Mahreen, K. (2020). Inequalities for a unified integral operator via $(\alpha, m)$-convex functions. Journal of Mathematics, 2020, Article ID 2345416, 9 pp.

[29] Farid, G. (2019). Estimations of Riemann-Liouville $k$-fractional integrals via convex functions. Acta et Commentationes Universitatis Tartuensis de Mathematica, 23(1), 71-78.

[30] Chen, Z., Farid, G., Rehman, A. U., \& Latif, N. (2020). Estimation of fractional integral operators via convex functions and related results. Advances Difference Equations, 2020, Article No 163.

[31] Farid, G. (2021). Bounds of Riemann-Liouville fractional integral operators. Computational Methods for Differential Equations, 10.22034/cmde.2020.32653.1516, In Press.

[32] Farid, G. (2019). Some Riemann-Liouville fractional integral inequalities for convex functions. The Journal of Analysis, 27(4), 1095-1102.

[33] Farid, G., Nazeer, W., Saleem, M. S., Mehmood, S., \& Kang, S. M. (2018). Bounds of Riemann-Liouville fractional integrals in general form via convex functions and their applications. Mathematics, 6(11), Article No 248.

[34] Chen, L., Farid, G., But, S. I., \& Akbar, S. B. (2021). Boundedness of fractional integral operators containing Mittag-Leffler functions. Turkish Journal Of Mathematics, To Appear.

[35] Kwun, Y. C., Farid, G., Kang, S. M., Bangash, B. K., \& Ullah, S. (2020). Derivation of bounds of several kinds of operators via $(s, m)$-convexity. Advances in Difference Equations, 2020, Article No 5. 
[36] Farid, G., Akbar, S. B., Rehman, S. U., \& Pečarić, J. (2020). Boundedness of fractional integral operators containing Mittag-Leffler functions via $(s, m)$-convexity. AIMS Mathematics, 5(2), 966-978.

() 2021 by the authors; licensee PSRP, Lahore, Pakistan. This article is an open access article distributed under the terms and conditions of the Creative Commons Attribution (CC-BY) license (http://creativecommons.org/licenses/by/4.0/). 